\title{
98. Radiation Response of Brain Tumor in Man
}

\author{
Chikao Izumi \\ Neturosurgical Clinic, 2nd Tokyo National Hospital \\ Masayuki WaTANABE \\ Surgical Clinic, 2nd Tokyo National Hospital \\ Yonosuke YAMAZAKI \\ Div. of Anesthesiology, 2nd Tokyo National Hospital
}

Eiichi Sugaya and Yoshio Karahashi

1st Dept. of Physiology, Kanagawa Dental College

Direct Cortical Response (DCR) and Radiation Response (RR) in animal have been extensively studied, but little is known about those of brain tumor in man. The authers have recorded RR during neurosurgical operation of brain tumor and following results were obtained.

1) When the tumor was situated near the cortical surface, the RR is not detectable in the overlaying cortex even when the cortical surface has no change in appearance.

2) In the case of deep situated tumor, no remarkable change was seen in RR.

\section{Case Reports of Subdural Abscess \\ Morio SaIto, Yoshihisa Onodera, Toshitsugu MaKI, Kunihiko TaKanashi and Tetsuro HIWA \\ Department of Neurosurgery, Tokyo Medical College Hospital}

Five cases of subdural abscess with characteristic clinical findings have been studied in our clinic for two year.

It has been recognized that subdural abscess recently has a tendency to increase. It was considered that these subdural abscess were caused by one sinuitis, two otitis media, one facial abscess, one infectious subdural heamatoma.

The localization of the abscess were investigated by operation and autopsy. There were confirmed three convexity hemishere, one parasagital and one bilateral convexity.

Symptom is due to the time from onset and is died into meningial irritation, intracranial hypertension and focal symptom. Concerning focal symptom, lesion localized on the convexity showed hemiplegia and convulsion, and parasagital lesion revealed monoplegia of lower extremity.

$$
-180-
$$


Cerebroangiography is of value in diagnosis. Avascular zone of anteriorposterior picture in convexity lesion, "Randbildende Gefässe" in parasagital lesion were shown in cerebroangiographie. Liquor of the lumbar puncture were within normal level except two cases of so called aseptic inflammation.

Therapy and comment will be discussed.

\title{
100. Clinical Survey of 153 Consecutive Cases of Cerebral Arachnoiditis; The Effect of Combinaiton of Spinal Tap with Instillation of Air and Steroid Hormon for Ophthalmologic Symptoms
}

\author{
Tetusya ShIRao, Hitoshi IchIKI, Kazuo UchiYama, Yoshiaki Okuma \\ Kennosuke TAKezaKo and Akira SaKamoto \\ 1st Department of Surgery, Kagoshima University School of Medicine
}

In the Ist Department of Surgery, Kagoshima University School of Medicine, 153 patients of cerebral arachnoiditis, 115 non-traumatic and 38 traumatic cases, were investigated. Symptoms of eyes which patients complained were as follows; diminution of vision, contraction of the visual fields, diplopia, blepharoptosis, photophobia and pain of eyes.

As the treatment, spinal tap with instillation of air, blocking of stellate ganglion, steroid hormon and their combinations were performed. In general, 75\% of these patients seemed to have clinical improvement: visual acuity were improved in $53 \%$, visual fields in $65 \%$, diplopia in $71 \%$, ptosis in $84 \%$, and pain of eyes in $80 \%$. As various methods and combination of treatments as described above have been evaluated, the most effective treatment was combination of all three methods.

101. Two Cases, One with Craniopharyngioma-like Syndrome and the Other with Precocious Puberty, associated with Suprasellar Calcification after Tuberculous Meningitis

Tetsuya Leslie SASABE, Katsuhito AgaKI, Yuzuru OKU, Yukitaka Ushio and Nobufusa AoYama

Department of Neurological Surgery, Osaka National Hospital

(Case 1): A 15-year-old boy, a victim of tuberculous meningitis in his fourth year, had noted polyuria and a developmental disturbance since around the 